\title{
Frequent Human Genomic DNA Transduction Driven by LINE-1 Retrotransposition
}

\author{
Oxana K. Pickeral, ${ }^{1,2}$ Wojciech Makałowski, ${ }^{2}$ Mark S. Boguski, ${ }^{1,2}$ and Jef D. Boeke ${ }^{1}$ \\ ${ }^{1}$ Department of Molecular Biology and Genetics, Johns Hopkins University School of Medicine, Baltimore, Maryland 21205, \\ USA; ${ }^{2}$ National Center for Biotechnology Information, National Institutes of Health, Bethesda, Maryland 20894 USA
}

\begin{abstract}
Human L1 retrotransposons can produce DNA transduction events in which unique DNA segments downstream of $\mathrm{Ll}$ elements are mobilized as part of aberrant retrotransposition events. That Lls are capable of carrying out such a reaction in tissue culture cells was elegantly demonstrated. Using bioinformatic approaches to analyze the structures of $\mathrm{Ll}$ element target site duplications and flanking sequence features, we provide evidence suggesting that $\sim 15 \%$ of full-length $\mathrm{L} 1$ elements bear evidence of flanking DNA segment transduction. Extrapolating these findings to the 600,000 copies of $\mathrm{Ll}$ in the genome, we predict that the amount of DNA transduced by $\mathrm{Ll}$ represents $\sim 1 \%$ of the genome, a fraction comparable with that occupied by exons.
\end{abstract}

The LINE-1 (L1) retrotransposon family is estimated to contain 600,000 copies, accounting for at least $15 \%$ of the human genomic DNA (Smit 1996). L1's second ORF (ORF2) encodes endonuclease and reverse transcriptase activities (Mathias et al. 1991; Feng et al. 1996), and is the most abundant ORF in the genome. As a major source of reverse transcriptase, L1 is likely to be indirectly responsible for the spreading of other retrotranscripts, such as Alu sequences and processed pseudogenes (Maestre et al. 1995; Boeke and Stoye 1997; Dhellin et al. 1997; Jurka 1997). A novel feature of L1 propagation and function was described recently by Moran et al. (1999), who showed that L1 can efficiently comobilize a 3 '- flanking segment of non-L1 DNA to new genomic locations in tissue culture cells. This makes L1 a potential player in such genomic events as exon shuffling and regulatory region combinatorics (Boeke and Pickeral 1999; Eickbush 1999). We have studied 129 full-length L1 elements with high similarity to L1.2 (an active element). Computational analysis shows that at least $10 \%$ of these L1s have an associated putative 3 '-transduced segment, and on this basis, the total amount of DNA transduced by L1 can be extrapolated to represent at least $1 \%$ of the human genome. This finding demonstrates that L1s are often involved in shuffling genomic DNA, and are thus important contributors to genome plasticity.

Several examples of naturally occurring $3^{\prime}$ transduction events were identified previously as mutagenic L1 insertions, in which additional (non-L1) sequences were incorporated downstream from each newly transposed L1 (Miki et al. 1992; Holmes et al. 1994; McNaughton et al. 1997). Transduction of 3'flanking sequence by engineered L1 elements also

\footnotetext{
'Corresponding author.
}

E-MAIL jboeke@jhmi.edu; FAX (410) 614-2987. readily occurs in HeLa cells, and can be driven by either the cytomegalovirus promoter, or the native L1 promoter; notably, transposition efficiency is higher when a strong polyadenylation signal is introduced downstream from the L1 (Moran et al. 1999). An important open question remains-how efficiently does L1driven 3' transduction occur naturally in the human genome? Opportunities to observe abnormal human phenotypes caused by 3 '-transducing L1 insertions may be extremely limited because only a small fraction of the human genome is currently attributed to genes and upstream regulatory regions. In this study, we took advantage of the tremendous sequence production by the Human Genome Project to computationally estimate the extent of naturally occurring L1-driven 3' transduction (Fig.1).

Full-length L1 elements are $\sim 6000$ bp long; the majority of L1s in the human genome, however, are severely $5^{\prime}$ truncated or rearranged, including 5'-inverted and deleted-inverted forms (Hutchison et al. 1989). Newly inserted L1 sequences are frequently flanked by short direct repeats, which have been shown to represent target site duplications (TSDs) created upon L1 integration (Kazazian et al. 1988; Holmes et al. 1994; Moran et al. 1996). With these sequence features in mind, we designed a TSD-based strategy to look for potential 3'-transduced segments associated with fulllength L1s (see Methods). If a pair of TSDs is found immediately flanking the L1 and its poly(A) tail, this represents a standard L1 insertion, with no additional sequences transposed. In contrast, in cases of 3' transduction the $3^{\prime}$ TSD is found further downstream from the L1 (Miki et al. 1992; Holmes et al. 1994; McNaughton et al. 1997).

\section{RESULTS AND DISCUSSION}

We limited our present study to full-size L1s with high 


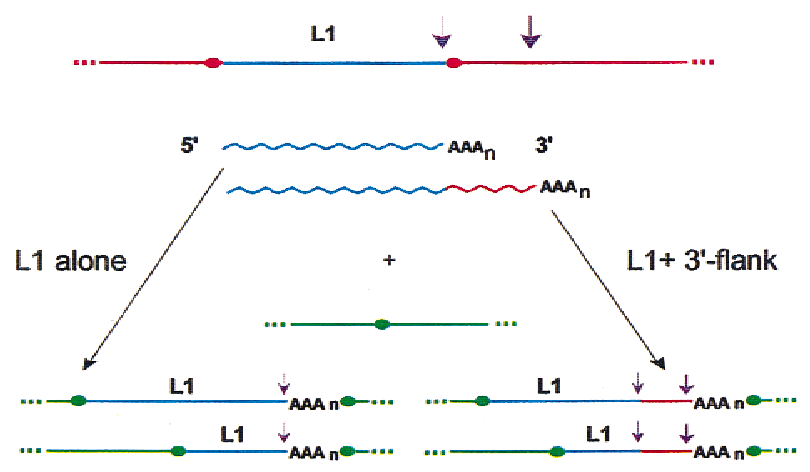

Figure 1 L1-driven 3' transduction. Incorporation of additional ( $3^{\prime}$ flanking) sequence into the transcript generated from the L1 promoter is followed by reverse transcription and integration of this longer cDNA into new genomic loci. (Ovals) Target site duplications; (purple arrows) polyadenylation signals (weak and strong); $\left(\mathrm{AAA}_{n}\right)$ poly $(\mathrm{A})$ tail.

similarity ( $>94 \%$ identity) to L1.2. Studying whole insertion events is critical for the TSD-based algorithm for 3 '-transduction detection, and this allows us to avoid introducing the extra ambiguity of the precise $5^{\prime}$ boundaries of each L1, which is a prominent factor when truncated elements are analyzed. We studied 129 full-length L1 elements; of these, 16 were uninformative because of insufficient flanking sequence in the GenBank records. An additional 16 examples lacked TSDs $>6$ bp in length. Another 76 cases represented

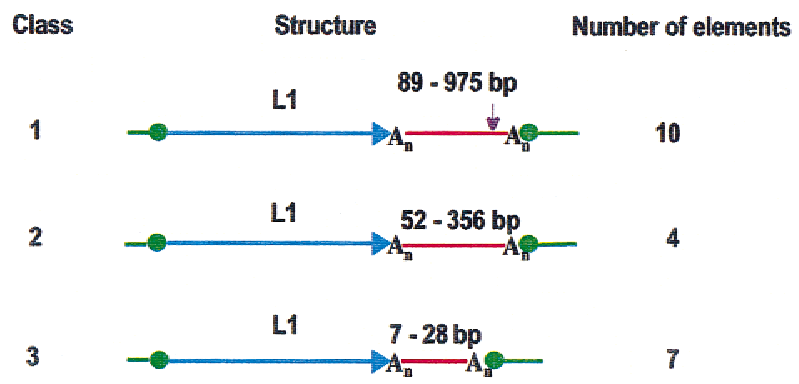

Figure 2 Three classes of L1 elements with 3' transduced segments. Transduced segments are shown in red. Range of transduced segment lengths is shown above each line. (Ovals) Target side duplications; (purple arrow) polyadenylation signal found in the transduced regions of class $1 ;\left(A_{n}\right)$ poly $(A)$ tail.

standard insertions. Finally, 21 qualified as $3^{\prime}$ transduction candidates. These 21 elements could be divided into three classes on the basis of sequence characteristics of the transduced DNA segment (Fig. 2; Table 1). Class 1 elements had downstream segments 89-975-bp long, and contained a consensus polyadenylation signal (AATAAA or ATTAAA) (Tabaska and Zhang 1999) 10-35 bp upstream from the poly(A) tail immediately preceding the $3^{\prime}$ TSD. The 10 elements in this class are the most likely candidates for $3^{\prime}$ transduction. Class 2 elements had 3' segments 52-356-bp long, and lacked a consensus polyadenylation signal. Class 3 segments are shorter than the other two classes

Table 1. Summary of L1-Associated 3' Transduction Events

\begin{tabular}{|c|c|c|c|c|c|c|c|c|c|}
\hline \multirow[b]{2}{*}{$\begin{array}{l}\text { L1 element } \\
\text { (gi no. and coordinates) }\end{array}$} & \multicolumn{4}{|c|}{ L1 structure ${ }^{a}$} & \multirow[b]{2}{*}{ Class } & \multirow[b]{2}{*}{ TSDs } & \multirow[b]{2}{*}{$\begin{array}{c}\mathrm{pA} \\
\text { signal }\end{array}$} & \multirow[b]{2}{*}{$\begin{array}{l}\text { Poly(A) } \\
\text { tail (bp) }\end{array}$} & \multirow{2}{*}{$\begin{array}{l}\text { Transduced } \\
\text { segment } \\
\text { length (bp) }\end{array}$} \\
\hline & $\begin{array}{l}\text { length } \\
\text { (bp) }\end{array}$ & ORF1 & ORF2 & $\begin{array}{l}\text { poly(A) } \\
\text { tail (bp) }\end{array}$ & & & & & \\
\hline $1279499,11400 \ldots 17418$ & 6019 & - & - & 25 & 1 & AAGAAAGAGCCAA & АТTAAA & 27 & 479 \\
\hline $1669380,(16606 \ldots 22620)$ & 6015 & - & - & 24 & 1 & AGAAATTGTC & ATTAAA & 17 & 89 \\
\hline $2275172,(73720 \ldots 79740)$ & 6021 & + & - & 22 & 1 & AGAAATCTGATTTGA & AATAAA & 13 & 975 \\
\hline 2588618, (59187 . 65202) & 6016 & + & - & 27 & 1 & GAAATTTTTCATC & AATAAA & 12 & 337 \\
\hline 2588627, $131175 \ldots 137191$ & 6017 & - & + & 14 & 1 & AАAAATTTACTGTCTA & AATAAA & 10 & 455 \\
\hline 2809267, (11716 . . 17735) & 6020 & + & + & 47 & 1 & AAAAAAAATCACCA & AATAAA & 15 & 137 \\
\hline $2853183,4261 \ldots 10276$ & 6016 & + & + & 36 & 1 & AAAGAAACTGAAGAGG & AATAAA & 25 & 175 \\
\hline $3319674,61629 \ldots 67639$ & 6011 & + & - & 16 & 1 & AAAGAAAAAATG & AATAAA & 17 & 272 \\
\hline $3810569,21041 \ldots 27057$ & 6017 & + & - & 24 & 1 & AGAAGCA & ATTAAA & 14 & 821 \\
\hline $3845408,(22572 \ldots$ 28582) & 6011 & - & - & 17 & 1 & AGAATCTGACСТTCC & AATAAA & 13 & 460 \\
\hline $2076718,29372 \ldots 35393$ & 6022 & - & - & 18 & 2 & AAGAAAGAGAATA & - & 9 & 356 \\
\hline $3288437,65129 \ldots 71148$ & 6020 & + & - & 16 & 2 & AAAGAACACCTGGG & - & 11 & 52 \\
\hline 3522964, (24909 . . 30926) & 6018 & + & + & 61 & 2 & AAAAAACACAGTGAA & - & 14 & 119 \\
\hline $4006838,117572 \ldots 123596$ & 6025 & + & - & 28 & 2 & AGAAGAAATG & - & 22 & 36 \\
\hline $3421083,114651 \ldots 120654$ & 6004 & - & - & 37 & 3 & AATGTTTA & AATAAA & 28 & 16 \\
\hline $3510243,(4788 \ldots 10794)$ & 6007 & - & - & 16 & 3 & AAATAGT[T/C]GAAGA & AATAAA & 21 & 7 \\
\hline $3808075,6601 \ldots 12628$ & 6028 & + & + & 23 & 3 & AAAAAGGAGCCCGG & AATAAA & 18 & 11 \\
\hline $3873180,114223 \ldots 120240$ & 6018 & + & - & 26 & 3 & AAGATTTTGTG & AATAAA & 26 & 10 \\
\hline $3928116,61076 \ldots 67082$ & 6007 & - & - & 18 & 3 & AGAAACTTGGAACACA & AATAAA & 6 & 8 \\
\hline $3953485,108923 \ldots 114934$ & 6012 & - & - & 31 & 3 & AAGCAATTTGT & - & 17 & 26 \\
\hline $4107187,91010 \ldots 97031$ & 6022 & - & - & 9 & 3 & AAACAGTATTTTCССТ & AATAAA & 8 & 13 \\
\hline
\end{tabular}


(7-26 bp), and could represent aberrant poly(A) tails. Even though class 3 segments may well have been formed by the same mechanism, further sequence analysis was not pursued because the results would not be statistically significant.

We then searched for a possible origin of the 14 elements of classes 1 and 2 and their $3^{\prime}$ transduced segments. Each of the potential transduced segments was masked by RepeatMasker (A. Smit and P. Green, unpubl.) to suppress highly repetitive matches, and the masked sequences were then used as queries in BLASTN and BLASTX similarity searches. A highscoring matching segment elsewhere in the genome, if found immediately downstream from another L1 element, would represent a potential master element if no poly(A) tail followed the segment of interest, or a related 3 ' transduction event if another poly(A) tail with target site duplications were found.

Of the fourteen 3 '-transduced segments, three were completely masked by RepeatMasker, and four were partially masked. Three of the four L1s with partially masked 3 '-transduced segments represent a previous integration into an L1 3' UTR, and one represents integration into an $A l u$ repeat. Interestingly, all of these L1 elements inserted in the same orientation as the target element (Fig. 3), which may be indicative of an L1 targeting preference. The TSD-based algorithm allows one to distinguish a transduction event with inclusion of repetitive sequence present at the master locus from a new L1 insertion into a pre-existing L1 3' UTR: In each case in Figure 3, a full-length, uninterrupted L1 is followed by the poly(A) tail and the sequence of interest (including other transposon sequence), followed by another poly(A) tail. All of this inserted material is flanked by TSDs. This sequence signature is consistent with a 3 '-transduction event in which the master L1 that produced each insertion had inserted previously into another transposon (see Fig. 3 legend for details). These events also suggest a simple mechanism for the rapid evolution of L1 (and other retrotransposon) 3' ends, which are structurally quite diverse.

Several of the unique sequences present in $3^{\prime}$ transduced segments produced significant nucleotide matches, whereas no protein matches (representing potential coding exons) were found. Twelve of the fourteen segments did, however, contain at least one ORF $>50$ bp long: two were $<80$ bp long, four between 80 and 100 bp long, and six were $>100$ bp long. Six of the fourteen segments associated with L1s of classes 1 and 2 (identified in the text by the gi numbers of their GenBank records-see Table 1) produced significant non-self nucleotide matches in the human nonredundant database. In three cases, gi2076718, 2853183, and 3522964 , no L1 was found upstream from the matches. Sequence gi2275172 represents an example of 3' trans-

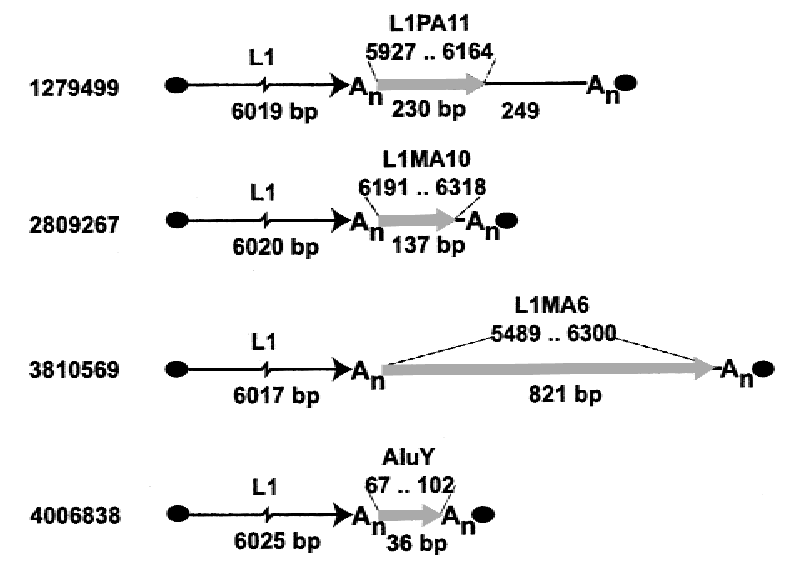

Figure 3 L1 integration into other transposons. Numbers at left indicate gi numbers of GenBank records in which the L1 elements and their associated $3^{\prime}$-transduced segments are found (see Table 1 for exact coordinates). Repetitive elements recognized in the 3 '-transduced segments by RepeatMasker are represented by filled gray arrows, with annotation shown at top (L1 or Alu subfamily name is followed by the coordinates projected onto the consensus sequence for that subfamily). Remarkably, in all four cases, the L1 that produced the observed new insertion depicted here must have inserted previously into another L1 or Alu in the same orientation. [A similar tendency has been noticed previously for newly inserted Alu elements (Jurka 1995)]. In two of the examples shown above, the transduced segment terminates at the end of the pre-existing L1. This suggests that once an L1 element inserts into such a region, subsequent transcription of the newly inserted element frequently reads through into the flanking DNA, and is then polyadenylated using a signal in the $3^{\prime}$ UTR of the pre-existing L1 element. By this means, L1 elements may acquire new, hybrid 3' UTRs. This process likely contributes to the rapid evolution of L1 3' UTR sequences (Smit 1996) and could explain why they are so rich in $A$ residues, as well as provide a clue to the mechanism leading to the similarity of L1 and Alu 3' UTR sequences (Boeke 1997).

duction we expected to find as a positive control-this case is a previously described L1-driven 3 '-transduction event in the human dystrophin gene (McNaughton et al. 1997). The last two of the query segments, gi2588627 and 3288437, shared a unique 31-bp sequence immediately following L1. Further analysis of the DNA flanking each of these two elements suggests that they are several transposition events removed from the same master element, which acquired additional flanking sequences in several steps (Fig. 4).

Of 113 informative L1 elements in the data set studied here, 97 (86\%) had TSDs consistent with either a standard insertion or a 3'-transduction event. Of these 97 L1s, 10\% (class 1 elements) are excellent candidates for 3 ' transduction, whereas 14\%-22\% have a 3 '-transduced segment by more relaxed criteria (including classes 2 and 3). Importantly, known and new examples of related 3 '-transduction events were identified. To estimate the total fraction of the human genome that can be accounted for by 3 ' transduction, we assumed $\sim 600,000$ copies of L1 in the human genome (Smit 1996) and the average length of transduced seg- 


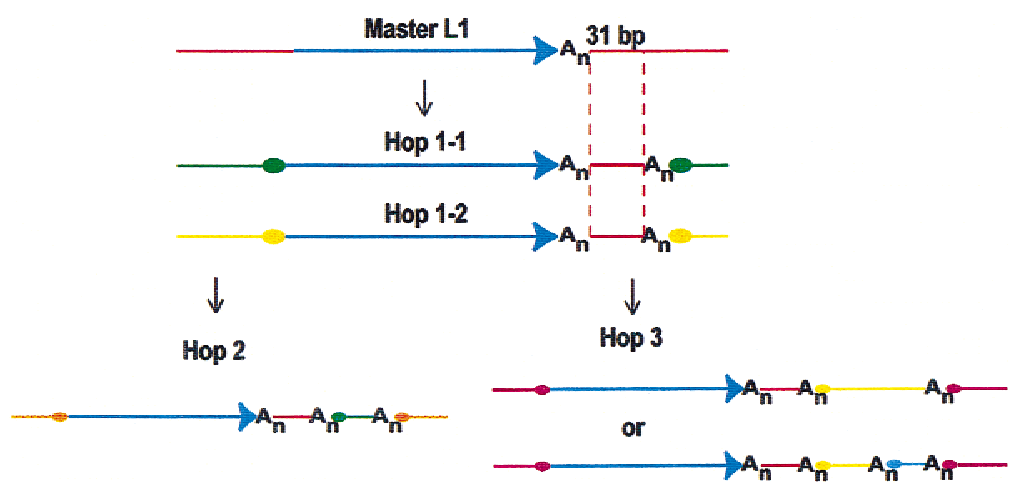

Figure 4 Model for the generation of L1 elements and their 3'-transduced regions in gi 3288437 and gi 2588627. Hop 2 (gi 3288437) and Hop3 (gi 2588627) are likely to be related descendants of the same Master L1 element. At least two, and possibly three (in case of gi 2588627) 3'-transducing intermediate transposition events would result in the structures seen at the DNA sequence level. The 31 -bp sequence immediately downstream from the L1 is 100\% identical between the two transduced segments, and in both cases is followed by a poly(A) tail. The proposed intermediates have not yet been found in the human genomic sequence available to date. The blue segment in Hop 3 reflects the possibility of two rather than one intermediate retrotransposition events between the master and Hop 3 elements, as there are two internal poly $(\mathrm{A})$ sequences within the transduced segment.

ments 420 bp for class 1 elements, $340 \mathrm{bp}$ for classes 1 and 2 combined, and $231 \mathrm{bp}$ for all 3 classes of transducing L1s. By use of these values, the total amount of 3 '-transduced sequence shuffling extrapolated to the entire human genome is between 25.2 and $30.5 \mathrm{Mb}$, or $\sim 1 \%$ of the human genome, a fraction comparable with that occupied by exons.

The estimate of L1 numbers on which the above calculation is based, was extrapolated from the amount of repeat DNA found in various large DNA clones selected from the human genome for sequencing. There is some ambiguity in the number of L1s in the human genome, mainly due to the difficulty of recognizing old elements in the sequence. In addition, our current knowledge of highly heterochromatic and potentially difficult to clone regions is limited; these are likely to be enriched in transposon sequences. Balancing this, the L1s without TSDs may result from either 3'transduction events of very large segments or from very short TSDs, thus the actual frequency of transduction events may be higher than our estimate. Finally, because L1s tend to localize to less gene-rich regions, the chances of carrying an exon may be decreased.

A final issue regarding the above estimate is the validity of extrapolating our numbers to the very large class of 5 '-truncated L1 elements. We expect that they will have as high a number of associated transduced segments as full-length elements, if not higher. We carried out a pilot survey of 25 randomly selected 1001000-bp long 5'-truncated L1 elements. These had characteristics similar to the full-length L1 elements in this study. The truncated elements analyzed were $85 \%-99 \%$ identical to L1.2 sequence, and of the 25 truncated L1 elements, 1 was uninformative (insufficient flanking sequence), 9 had no TSDs >7-bp long, and 15 had TSDs. Of the elements with TSDs, 11 were classified as standard insertion, and 4 represented a 3 '-transduction signature. Of these four candidates, two were completely masked (L1 3' UTR sequence, in the same orientation as the L1 of interest). This preliminary survey supports our expectation of finding an equivalent or slightly higher fraction of 3 ' transduction in truncated elements as opposed to full-length L1s.

A possible expansion of this study would be the study of older L1 subfamilies. However, this would bring a higher ambiguity level to the signals seen at the DNA sequence level. Elements of the young subfamilies are more likely to have inserted relatively recently, and thus would have a higher chance of preserving intact TSDs. The older an insertion, the lower the likelihood of finding authentic TSDs, because of the accumulation of random mutations.

Thus, L1-driven transduction of flanking DNA is likely to be an important mechanism of genome evolution via increasing genome plasticity and facilitating new combinations of coding and regulatory sequences. Although most observed cases of 3 ' transduction represent relatively small gene segments, it is possible that entire genes are included occasionally into transduced segments. This would lead to the introduction of a processed (no introns) copy of a gene into a new genomic locus. Thus, 3' transduction is potentially a mechanism for outright gene duplication as well as gene scrambling. A 3' transduction might represent a relatively noninvasive mechanism by which an organism can test novel sequence combinations, because transposon-plus-flanking-sequence integration could be significantly less disruptive to genome organization than larger-scale genome rearrangements such as inversions or translocations.

\section{METHODS}

\section{Data set}

A total of 129 L1 elements, at least 6000-bp long, were collected as high-scoring BLAST matches to L1.2 (accession no. M80343), one of the currently active LINE-1 transposons. The search was performed against GenBank release 113.0 plus daily updates as of September 1, 1999. All of these elements are at least $94 \%$ identical to L1.2.

\section{Target Site Duplication Determination}

One hundred base pairs upstream of each L1 element studied were compared with 3000 bp downstream of the same element, in search of short direct repeats that are the putative

\section{Genome Research}


TSDs. BLAST2SEQUENCES (Tatusova and Madden 1999) and DOTTER (Sonnhammer and Durbin 1995) computer programs were used to find matching substrings at least 6-bp long. One mismatch was allowed for repeats $>11$-bp long. A pair of short direct repeats was considered a TSD pair if the 5' TSD adjoined the $5^{\prime}$ end of the L1 element and the 3' TSD immediately followed a poly(A) tail.

\section{DNA Sequence Analysis}

The BLASTN (Altschul et al. 1997) program was used to search human-specific nucleotide databases for sequences similar to the putative transduced regions; BLASTX (Altschul et al. 1997) program was used to search for protein matches. We used the e-value cutoff of 0.1 in both BLASTN and BLASTX searches. REPEATMASKER (A. Smit and P. Green, unpubl.) was used to mask and characterize the transduced segments.

\section{Databases Used}

The nonredundant (nr) protein database was used in BLASTX searches. In BLASTN searches, four human-specific databases were used, nr, EST, GSS, and HTGS.

\section{ACKNOWLEDGMENTS}

We thank John Moran and Greg Cost for helpful discussions. Our work was supported in part by NIH grant CA16519 to J.D.B.

The publication costs of this article were defrayed in part by payment of page charges. This article must therefore be hereby marked "advertisement" in accordance with 18 USC section 1734 solely to indicate this fact.

\section{REFERENCES}

Altschul, S.F., T.L. Madden, A.A. Schaffer, J. Zhang, Z. Zhang, W. Miller, and D.J. Lipman. 1997. Gapped BLAST and PSI-BLAST: A new generation of protein database search programs. Nucleic Acids Res. 25: 3389-3402.

Boeke, J.D. 1997. LINEs and Alus-the polyA connection. Nat. Genet. 16: 6-7.

Boeke, J.D. and O.K. Pickeral. 1999. Retroshuffling the genomic deck. Nature 398: 108-109, 111.

Boeke, J.D. and J.P. Stoye. 1997. Retrotransposons, endogenous retroviruses, and the evolution of retroelements. In Retroviruses (ed. H. Varmus, S. Hughes, and J. Coffin), pp. 343-435. Cold Spring Harbor Laboratory, Cold Spring Harbor, NY.

Dhellin, O., J. Maestre, and T. Heidmann. 1997. Functional differences between the human LINE retrotransposon and retroviral reverse transcriptases for in vivo mRNA reverse transcription. EMBO J. 16: 6590-6602.

Eickbush, T. 1999. Exon shuffling in retrospect. Science 283: $1465-1467$.
Feng, Q., J.V. Moran, H.H. Kazazian, Jr., and J.D. Boeke. 1996. Human L1 retrotransposon encodes a conserved endonuclease required for retrotransposition. Cell 87: 905-916.

Holmes, S.E., B.A. Dombroski, C.M. Krebs, C.D. Boehm, and H.H. Kazazian, Jr. 1994. A new retrotransposable human L1 element from the LRE2 locus on chromosome 1q produces a chimaeric insertion. Nat. Genet. 7: 143-148.

Hutchison, C.A., S.C. Hardies, D.D. Loeb, W.R. Shehee, and M.H. Edgell 1989. Long interspersed repeated sequences in the eukaryotic genome. In Mobile DNA (ed. D.E. Berg and M.M. Howe), pp. 593-617. American Society for Microbiology, Washington, DC.

Jurka, J. 1995. Origin and evolution of Alu repetitive elements. In The impact of short interspersed elements (SINEs) on the host genome (ed. R. Maraia), pp. 25-41. R.G. Landes, Austin, TX.

- 1997. Sequence patterns indicate an enzymatic involvement in integration of mammalian retroposons. Proc. Natl. Acad. Sci. 94: $1872-1877$.

Kazazian, H.H., Jr., C. Wong, H. Youssoufian, A.F. Scott, D.G. Phillips, and S.E. Antonarakis. 1988. Haemophilia A resulting from de novo insertion of L1 sequences represents a novel mechanism for mutation in man. Nature 332: 164-166.

Maestre, J., T. Tchenio, O. Dhellin, and T. Heidmann. 1995. mRNA retroposition in human cells: Processed pseudogene formation. EMBO J. 14: 6333-6338.

Mathias, S.L., A.F. Scott, H.H. Kazazian, Jr., J.D. Boeke, and A. Gabriel. 1991. Reverse transcriptase encoded by a human transposable element. Science 254: 1808-1810.

McNaughton, J.C., G. Hughes, W.A. Jones, P.A. Stockwell, H.J. Klamut, and G.B. Petersen. 1997. The evolution of an intron: Analysis of a long, deletion-prone intron in the human dystrophin gene. Genomics 40: 294-304.

Miki, Y., I. Nishisho, A. Horii, Y. Miyoshi, J. Utsunomiya, K.W. Kinzler, B. Vogelstein, and Y. Nakamura. 1992. Disruption of the APC gene by a retrotransposal insertion of L1 sequence in a colon cancer. Cancer Res. 52: 643-645.

Moran, J.V., S.E. Holmes, T.P. Naas, R.J. DeBerardinis, J.D. Boeke, and H.H. Kazazian, Jr. 1996. High frequency retrotransposition in cultured mammalian cells. Cell 87: 917-927.

Moran, J.V., R.J. DeBerardinis, and H.H. Kazazian, Jr. 1999. Exon shuffling by L1 retrotransposition. Science 283: 1530-1534.

Smit, A.F. 1996. The origin of interspersed repeats in the human genome. Curr. Opin. Genet. Dev. 6: 743-748.

Sonnhammer, E.L. and R. Durbin. 1995. A dot-matrix program with dynamic threshold control suited for genomic DNA and protein sequence analysis. Gene 167: GC1-GC10.

Tabaska, J.E. and M.Q. Zhang. 1999. Detection of polyadenylation signals in human DNA sequences. Gene 231: 77-86.

Tatusova, T.A. and T.L. Madden. 1999. BLAST 2 Sequences, a new tool for comparing protein and nucleotide sequences. FEMS Microbiol. Lett. 174: 247-250.

Received December 14, 1999; accepted in revised form February 25, 2000.
Genome Research www.genome.org 


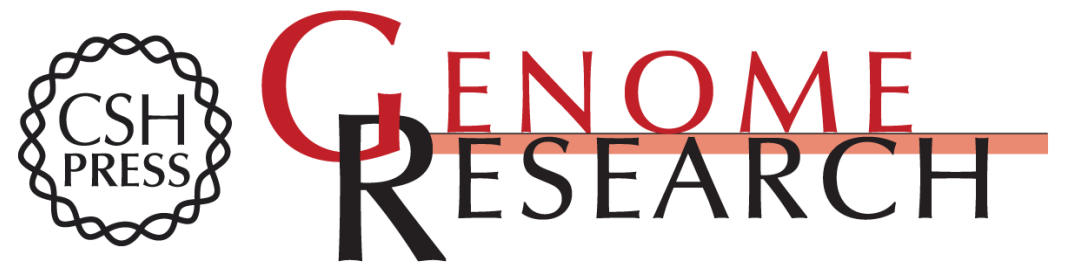

\section{Frequent Human Genomic DNA Transduction Driven by LINE-1 Retrotransposition}

Oxana K. Pickeral, Wojciech Makalowski, Mark S. Boguski, et al.

Genome Res. 2000 10: 411-415

Access the most recent version at doi:10.1101/gr.10.4.411

References This article cites 19 articles, 6 of which can be accessed free at:

http://genome.cshlp.org/content/10/4/411.full.html\#ref-list-1

\section{License}

Email Alerting Receive free email alerts when new articles cite this article - sign up in the box at the Service top right corner of the article or click here.

\section{Affordable, Accurate Sequencing.}

To subscribe to Genome Research go to: https://genome.cshlp.org/subscriptions 